\title{
Analysis of domestic and foreign research status of agricultural pollution control based on VOSviewer
}

\author{
Zhang Xin ${ }^{1, a}$, Zhao Keyu ${ }^{1, a}$, Peng $\operatorname{Lin}^{1, b^{*}}$ \\ ${ }^{1}$ College of big data, Yunnan agricultural university, Kunming, China \\ ${ }^{1}$ College of big data, Yunnan agricultural university, Kunming, China
}

\begin{abstract}
Visual analysis of the field of agricultural pollution prevention and control will help researchers to fully understand the research status at home and abroad, and better fit the current situation for further research. Mothod: Web of Science database was used to retrieve 2,214 literatures related to agricultural pollution prevention and control from 2000 to 2018 as data sources, and VOSviewer software was used for visual analysis. The results showed that the research heat of agricultural pollution control showed a good upward trend; Since 2010, China's rapid growth in the volume of publications has ranked first, while the United States has remained stable for a long time, ranking second; Chinese institutions hold eight of the top 10 spots, with the Chinese academy of sciences at the absolute center of the field; In the research hotspot, it is divided into three clusters.1\# In the field of agricultural pollution prevention and control, we have the ability to effectively control nitrogen, quality and phosphorus; China has made in-depth research on sediments, heavy metals and agricultural soils.3\# Indian researchers have paid considerable attention to the prevention and control of surface water, drainage basins and groundwater pollution, and carried out in-depth research work for this purpose.
\end{abstract}

\section{Introduction}

With the growing global population, the issue of food security is now a common problem facing the world. One of the main problems of food security is agricultural non-point source pollution. How to effectively prevent and control agricultural non-point source pollution is the key to solving food security. After China's industrial pollution and urban pollution have been effectively controlled, agricultural non-point source pollution has gradually become China's most important environmental problem [1]. Agricultural non-point source pollution will not only affect the water environment and soil environment, destroy agricultural ecosystems, endanger agricultural security, but also indirectly affect people's physical health and become a public hazard that harms national quality [1].

This research uses knowledge map visualization technology to show the field of agricultural non-point source pollution prevention and control. The visualization technology can show the development process, research status, frontier areas, and a multidisciplinary fusion of the overall knowledge framework of the discipline [2].

Using VOSviewer software for visual analysis, VOSviewer is a free mapping tool developed by Ludo Waltman and Nees Jan van Eck of Leiden University in the Netherlands, which is based on visualization technology to process the knowledge unit of literature [3]. VOSviewer can quickly analyze data when processing complex data, and presents a cluster network view with better results.

\section{Data source and method}

\subsection{Data source:}

In this study, a Web of Science database was used to search the literature published between 2000 and 2018, and the search formula was "TS = agricultural non-point source pollution prevention and control". The document type only extracted articles for refining to obtain 2214 document records as the data source.

\section{2 method:}

In this study, EXCEL was used as the auxiliary software to statistically analyze the publication volume, time, institution, and author distribution of the literature. Using VOSviewer visualization software to analyze the data source, the author's cooperation, institutional cooperation and co-occurrence clustering of keywords and the evolutionary path of research hot spots can be visualized.

a759572801@qq.com,* corresponding author: bdapengjiao@163.com 


\section{Literature publication distribution:}

According to table 2-1, we can get the number of circulated countries. The number of circulated countries in China during the 18 years reached 628, accounting for $29.71 \%$ of the total number of circulated papers. $23.89 \%$ of the total post volume. The third is the number of 146 papers published in England, accounting for $6.59 \%$ of the total (the proportion is not high). Based on the above data, it can be concluded that in the field of agricultural non-point source pollution prevention and control, China and the United States have reached $53 \%$ of the global publication rate, and have made important contributions to the field of global non-point source pollution prevention and control. The dominant position in this field is China, followed by the United States, which can also be obtained from Figure 1. The top 3 countries in the field of agricultural non-point source pollution prevention and control have close cooperation links, and they have many countries such as India, France, Canada, etc. There is cooperation. At the same time, it also shows that scientific researchers around the world are learning from the research results of high-yielding countries in this field for further research. The field of prevention and control of agricultural non-point source pollution is becoming more and more important, and it is also a common problem faced by countries in the process of agricultural production safety.

From Table, we can see the basic situation of the volume of papers issued by high-producing countries in the field of agricultural non-point source pollution prevention and control over the past years: China started late in this field. From 2000 to 2010, the number of papers increased from only 2 to 18 Steady growth trend. By the end of 2010, there was a rapid growth in the amount of circulated paper, and the volume of circulated paper surpassed the top of the US alone. It shows that China has gradually paid more attention to the field of agricultural non-point source pollution control since 2000, and its research intensity has gradually deepened, and it has gradually become a research hotspot for scientific researchers. After 2014, the volume of research and publication has shown a sharp upward trend, At the same time, the increase in the number of articles is also in line with the ruling party's idea of setting up and practicing the concept that clear waters and green mountains are mountains of gold and silver, and adhering to the basic state policy of conserving resources and protecting the environment [4]. Compared with the United States, the number of documents reached 18 in the early 2000s, and it is better than other countries. This is inseparable from the developed agriculture in the United States. The United States government attaches great importance to the prevention and control of agricultural non-point source pollution, and the research environment is relatively mature and maintains a steady growth trend. The number of posts in the lower rankings of England, Spain, and India also gradually increased, reflecting that the government has gradually begun to pay attention to the field of agricultural non-point source pollution control, and scientific researchers have gradually entered this field for research showing a good trend.

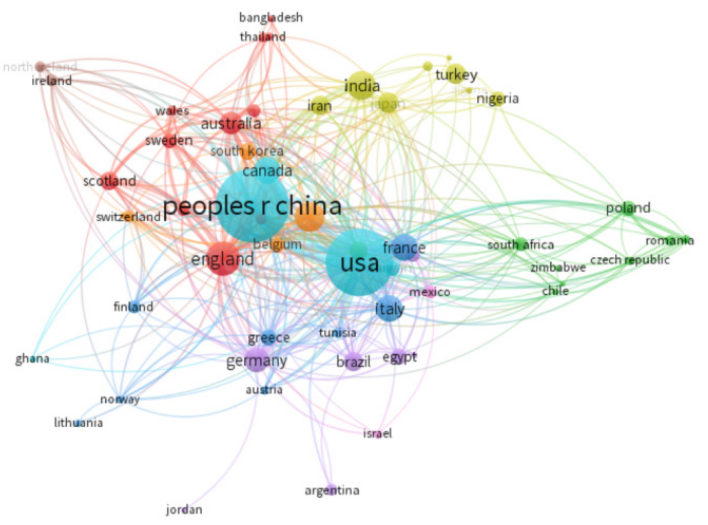

Figure1. Visualization of Cooperation among High-yielding Countries in the Field of Agricultural Pollution Control

TABLEI. THE TOP 5 COUNTRIES WITH HIGH OUTPUT IN THE FIELD OF AGRICULTURAL POLLUTION CONTROL HAVE PUBLISHED PAPERS OVER THE YEARS table 2-1

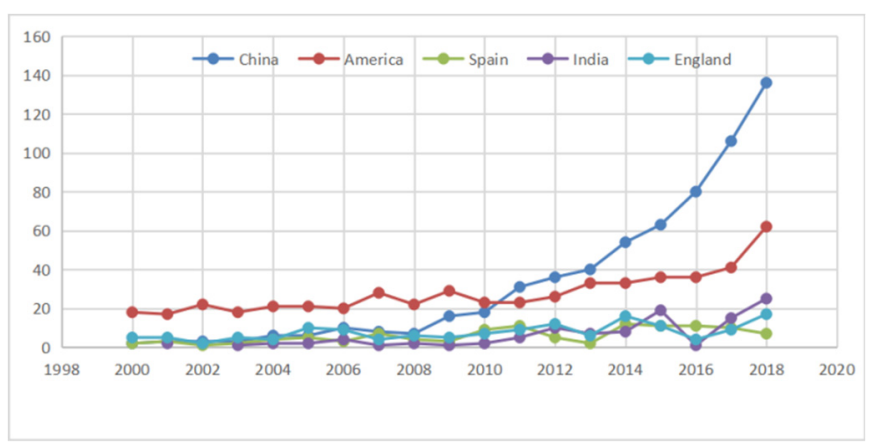

\section{Literature discipline distribution:}

Distinguishing the literature in the field of agricultural non-point source pollution control The disciplines that the literature belongs to contribute to the volume of publication of some disciplines, which can fully reflect the academic achievements, strong research strength, and high academic status of these disciplines, which are relatively strong compared to other disciplines. It is called strong discipline [5]. The field of agricultural non-point source pollution prevention and control was searched in the WOS database, and the number of pooled disciplines reached 104 disciplines. The following tableIIshows the details of the top 5 disciplines:

It can be clearly seen that the top 5 subjects in the discipline are: the first is environmental science (the number of records is: 1198 , the proportion is: $54.11 \%$ ); the second is water resources (the number of records is : 510, proportion: $23.035 \%$ ); third place is comprehensive earth science (number of records: 235, proportion: $10.614 \%$ ); fourth place is environmental engineering (number of records: 188 . The proportion is $8.491 \%$. The fifth place is ecology (the number of records is 167 and the proportion is $7.543 \%$ ). Since then, it can be clearly seen that in many disciplines, environmental science accounts for $54.11 \%$ in the area of agricultural non-point 
source pollution prevention and control, which is $31 \%$ more than the second place in water resources. It fully reflects the importance of environmental science in the field of agricultural non-point source pollution control, so it can be called a strong discipline in this field. In the field of agricultural non-point source pollution prevention and control, extended research is mainly aimed at environmental problems and environmental pollution problems. There is also a $23.035 \%$ ratio of water resources here, indicating that water resources also occupy a very important position in this field.

\section{Visual analysis of agency cooperation:}

The use of VOSviewer software to visually analyze the data downloaded by the WOS database helps to comprehensively understand the distribution and cooperation of core institutions in the field of agricultural non-point source pollution control. By analyzing and processing the data, the threshold of the minimum number of cooperation of the institution is adjusted to 5, and the cooperation map of the main research institutions in the field of agricultural non-point source pollution prevention and control is generated as shown in Figure 2.

\subsection{Organizational collaboration visualization:}

Each circle represents the name of a different institution, the size of the circle represents the degree of activity of the institution in the prevention of agricultural non-point source pollution, and the number of publications, and the color of the circle represents different clusters; the larger the circle, the larger the volume of the institution's publication. On the contrary, the smaller it is; the closer the two circles are, the closer the relationship is. Visual analysis of institutional cooperation using VOSviewer software on 2214 documents. As can be seen in Figure 2, there are 2,486 institutions in the world conducting research on agricultural non-point source pollution control. Among the top 10 high-yielding institutions are: Chinese Academy of Sciences241, USDA-ARS 58, China Agriculture University 33, Chinese environment sciences21, Beijing Normal University 48, Zhejiang university 37, Chinese Academy of Agricultural Sciences21, Purdue University 22, Peking university 16 . Among the top 10 high-yielding institutions, there are 8 Chinese scientific research institutions in the field of agricultural non-point source pollution control, while only 2 scientific research institutions in the United States have entered the top 10. This shows that China's research institution team Occupying a core position in this field, the most prominent research organization is that the Chinese Academy of Sciences has published 198 documents, which is equivalent to the total number of publications in the major producing countries of England, Spain, and India. Therefore, it is undeniable that China Scientific research team institutions in agricultural non-point source pollution prevention Domain has made a great contribution.

\subsection{Author collaboration visual analysis:}

According to Price's law to determine the minimum volume of core authors: $m=0.749 \times \sqrt{\mathrm{N}_{\max }} \quad$ [6], The value sought is 2.48 , Rounded value is 3 . By using VOSviewer software to analyze the collaborative visualization of authors, the threshold is adjusted to 3 , as shown in Figure 3:

It can be seen from Figure 3 that the authors who have met the conditions in the field of agricultural non-point source pollution prevention and control have 192 authors participating in the research in this field. You can see the basic information about author cooperation in the entire field. There are many small-scale groups in this field that are isolated on the edge of the entire visualization. In the picture, there are two large author cooperation groups at the center of the entire field. One is the most productive author, and the number of Hao Fan Hua posts has reached 10 . The cooperating group centered on him has 20 members. The other is a collaborative group of authors represented by Christie, peter. Because this group is the largest group in the entire field, the group is independently visualized. As shown in Figure 4, the number of Christie, peter author has reached 9 in the center of the view. The author spreads the community. The literature, which was mainly published around 2014, is the largest author cooperation group in the field of agricultural non-point source pollution prevention and control. The number of members has reached 26 and the total number of authors has reached 103. More precisely, in the two largest groups, it is clear that the vast majority of authors are from China, indicating that the core authors in this field are mainly from China.

TABLEII. Number and percentage of papers published by each discipline

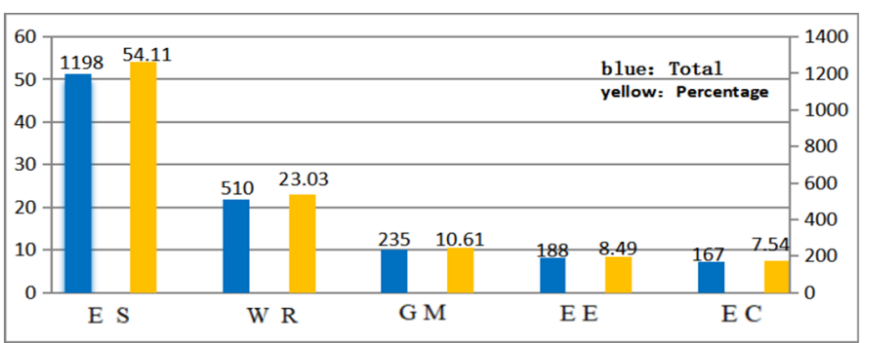

Note: ES- Environmental Sciences; WR- Water Resources; GM- Geosciences Multidisciplinary; EE- Engineering Environmental; EC- Ecology 


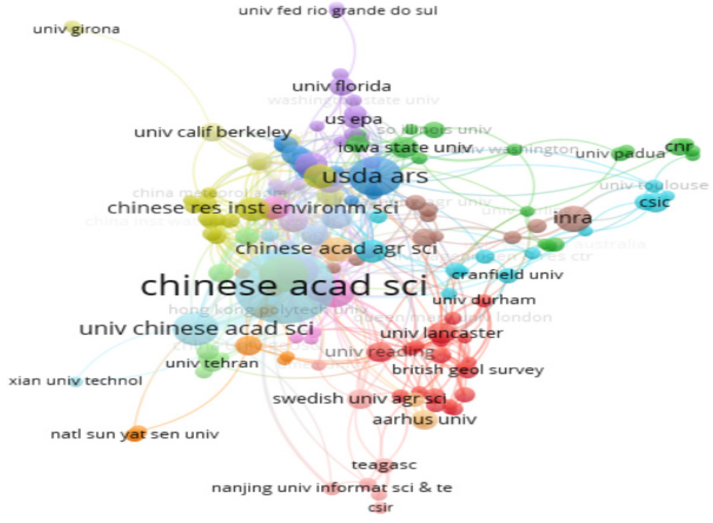

Figure2. Visualization of institutional cooperation in the field of agricultural pollution control

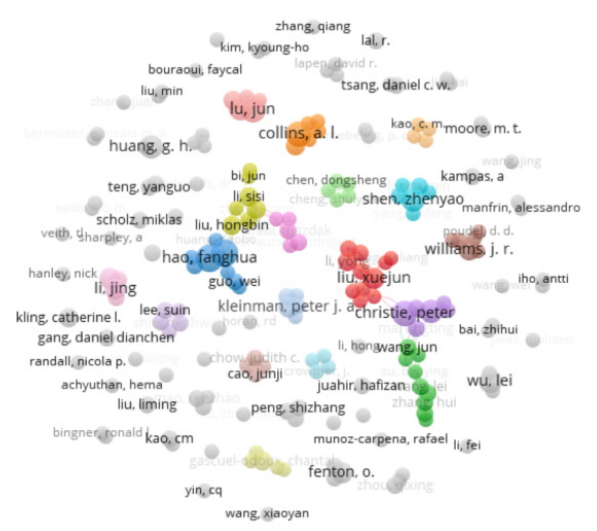

Figure3. Cooperative Visualization of Authors in the Field of Agricultural Pollution Control

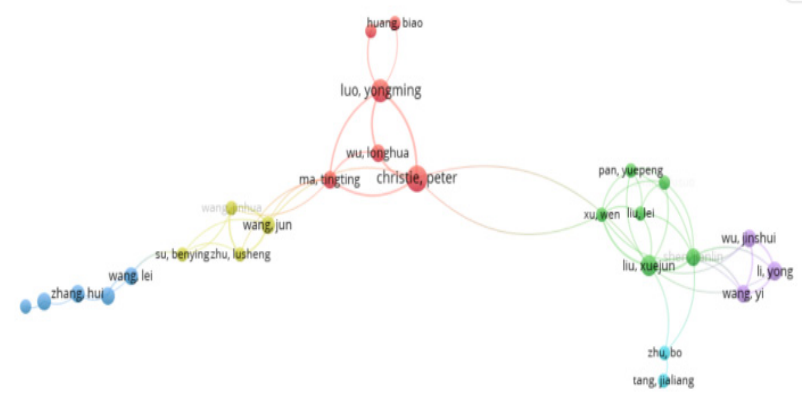

Figure4. Visualization of the largest cooperative group of authors in the field of agricultural pollution control

\section{Analysis of research hotspots in agricultural non-point source pollution prevention:}

Keywords are the author's refined summary of the content or research methods of the paper. Through analysis of high-frequency keyword co-occurrence maps, research hotspots in this field and evolution paths of research hotspots can be obtained [5]. In the WOS database, the agricultural non-point source pollution prevention and control field was searched, and 2214 pieces of data were retrieved (contamination and pollution merge, systems and system merge, etc.).
Keyword co-occurrence analysis was performed on the data to obtain 5608 keywords. The top 10 keywords for total occurrences are pollution365 times, soil203 times, management175 times, nitrogen 172 times, quality 167 times, water 160 times, sediment 140 times, phosphorus 136 times, agricultural soils 136 times, impact 132 times, and water-quality 122 times. It shows that the number of keywords co-occurrence is not high enough and there are fewer high-frequency words.

Adjust the minimum number of keywords co-occurrence to 30 to obtain a total of 63 keywords (as shown in Figure 5), and perform keyword density map analysis to obtain research hotspots in the field of agricultural non-point source pollution control. The main keywords are: pollution, soil, Management, nitrogen, quality, water, sediment, phosphorus, agricultural soils, impact, water-quality.

The minimum number of keywords co-occurrence is 30 to obtain a total of 63 keywords. Cluster analysis is performed to generate a keyword network co-occurrence view, as shown in Figure 6:

The generated keyword network analysis co-occurrence map is used to perform clustering to obtain three major categories, where each color represents a different cluster. Keywords included in cluster 1 \# (red) are: management, nitrogen, quality, phosphorus; keywords included in cluster 2 \# (cyan) are: pollution, sediment, china, heavy-metals, agricultural 28 keywords such as soils; Cluster 3 \# (blue) includes keywords: water, India, groundwater, and basin.

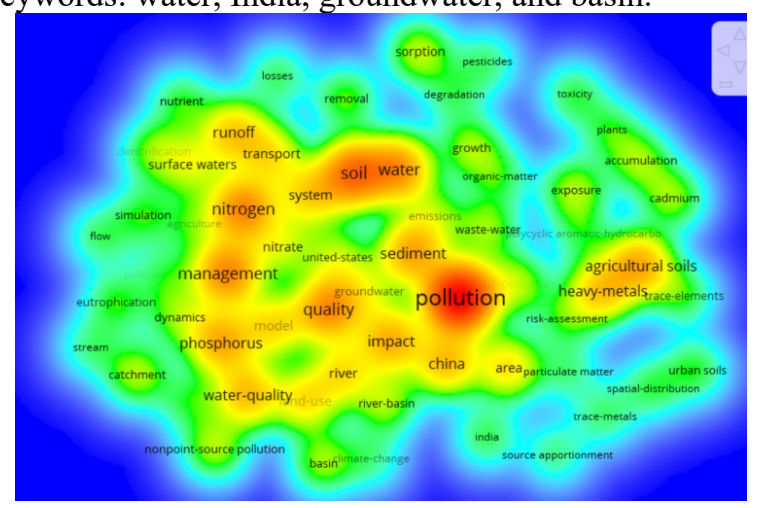

Figure5. Keyword density map

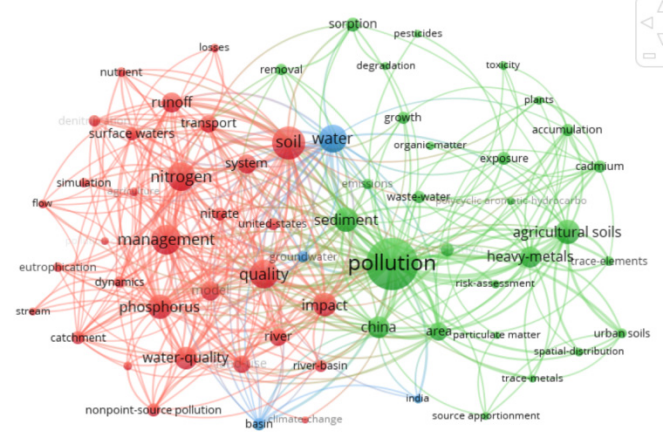

Figure6. Keyword network co-occurrence graph 


\section{7 in conclusion:}

This study visualizes the field of agricultural non-point source pollution prevention and control, and uses EXCEL for statistical analysis of disciplines in the field of agricultural non-point source pollution prevention and control. The authors and institutions perform network cooperation analysis. The frequency of keywords co-occurrence is adjusted. Finally a more reasonable density map and network co-occurrence map are obtained.

Conclusion: from the perspective of the total volume of papers issued worldwide, the agricultural non-point source pollution prevention and control field shows a rapid upward trend. Especially in China, which has the largest number of papers, although it started late, after 2010, the volume of papers surpassed that of the United States, which ranked first in the field. This shows that China has gradually paid more attention to the field of agricultural non-point source pollution prevention and control since 2000, and its research intensity has continued to deepen, which has led to a continuous increase in research results.

The main disciplines involved in the prevention and control of agricultural non-point source pollution are environment, water resources, integrated earth sciences, engineering environment, ecology, etc. The strong disciplines in the field of agricultural pollution prevention and control are environment and water resources, which account for the entire volume.

In the institutional cooperation visualization, 2486 institutions researched the field of agricultural non-point source pollution prevention. The main research institution China occupied 8 scientific research institutions as the absolute core position in this field. In the author's cooperation visualization, the entire agricultural non-point source pollution prevention field 8959 authors participated in the research in this field. Although the number of authors' cooperation is large, they are basically in a state of single-handedness. There are more 2-4 small-scale cooperations, which indicates that the lack of author-cooperative relationships in this field requires strengthened cooperation.

The keywords are analyzed to generate a keyword density map. From the density map, we can find that the current main research hotspots are: pollution, soil, management, nitrogen, quality, water, sediment, phosphorus, agricultural soils, impact, water-quality. The cluster analysis of the keywords shows that the three clusters are: cluster 1 \# (red). In this cluster, we can understand that there are nitrogen, In terms of quality, phosphorus, etc., it has better processing and prevention capabilities. Cluster 2 \# (cyan) In this cluster, we can understand that China's research hotspot are based on the environmental problems faced by the country at the current stage. In the targeted research, agricultural non-point source pollution has become an important research area. From the research hotspots, it can be concluded that China has in-depth research results in specific aspects such as sediments, heavy metals, and agricultural soils. The hotspot words clustered by cluster 3 \# (blue) are: water, India, groundwater, and basin.
Keywords: India's forests are largely reclaimed due to the imbalance between population and land, and large amounts of chemical fertilizers and chemical pesticides are used to achieve a short-term increase in grain yield, resulting in the destruction of the ecological environment, surface water and residents' reference water being polluted to varying degrees. As early as 1974, India established the Water Pollution Control Act to address water pollution problems. Therefore, Indian researchers pay more attention to surface water, watershed and groundwater, and have carried out in-depth research work for this purpose.

To sum up, the world has a good development trend in the field of agricultural non-point source pollution control. The large number of participating countries and scientific research teams shows that the field of agricultural non-point source pollution control is not just a problem faced by individual countries, but a common problem facing the world problem. The authors need to gradually strengthen cooperation when the authors cooperate less frequently and small groups are more frequent. Although there are many research points, there are still many unresolved pollution problems in the future agricultural development. Therefore, research groups and individuals around the world must not only strengthen the breadth of research in the field of agricultural pollution prevention, but also strengthen the in-depth research.

\section{Acknowledgment}

The findings and the opinions are partially supported by projects of the National Natural Science Foundation of China (NSFC), No. 31960290.This work is supported by Engineering and Technology Research Center for Yunnan Agricultural Big Data and Yunnan Agricultural University, China.

\section{References}

1. Zheng Xiang, "Economic Policy Evaluation of Agricultural Pollution Control in China,". Guangdong: Master's Degree Thesis of Guangdong Academy of Social Sciences, 2014.

2. Qin Chang Jiang, and Hou Han Qing. "Knowledge Map-A New Field of Information Management and Knowledge Management," Journal of Academic Libraries. vol.1, pp. 30-37,2009.

3. VOSviewer Visualizing scientific landscapes [EB / OL]. (2015-10-12) [2017-01-08]. http: //www.VOSviewer.com.

4. Yan Yan, and Zhao Jing. "Xi Jinping's report at the 19th National Congress of the Communist Party of China". People's Daily, October2017.

5. $\mathrm{Ma} \mathrm{Xu} \mathrm{Bu}$, and Liu Wei, and Qin Chun Xiu. "Analysis of the subject, knowledge base, research hotspots and evolution of policy evaluation in China based on knowledge map,". Modern Information, vol.39, pp.166-177,2019. 
6. Hou Hai Yan, and Guo Fang Qi, and Sun Tai An, and $\mathrm{Hu} \mathrm{Zi}$ Gang. "Analysis of domestic and international research status of Shandong Province's biotechnology based on VOSviewer,". Science and Management, vol.32, pp. 26-33, 2018. 